\title{
HXR photospheric footprints
}

\author{
J. C. Martínez-Oliveros, A.-C. Donea and P. S. Cally \\ Centre for Stellar and Planetary Astrophysics, School of Mathematical Sciences \\ Monash University, VIC 3800, Australia \\ email:Juan.Oliveros@sci.monash.edu.au
}

\begin{abstract}
We have analysed the $6 \mathrm{mHz}$ egression power signatures of some accoustically active $\mathrm{X}$-class solar flares. During the impulsive phase these flares produced conspicuous seismic signatures which have kernel-like structures, mostly aligned with the neutral line of the host active region. The kernel-like structures show the effect of constructive interference of the acoustic waves emanating from the complex sources, suggesting motion of the acoustic sources. The coaligment between the seismic signatures and the hard X-ray emission observed by RHESSI from the footpoints of the coronal loops suggests a direct link between relativistic particles accelerated during the flare and the hydrodynamic response of the photosphere during flares.
\end{abstract}

Keywords. Sun: X-rays, Sun: particle acceleration, Sun: helioseismology, Sun: sun quakes, Sun: solar flares

\section{Introduction}

Some solar flares emit strong acoustic transients into the solar interior during their impulsive phase (Kosovichev \& Zharkova 1995, 1998). These transients penetrate thousands of kilometers beneath the active region photosphere. They produce a characteristic signature tens of thousands of kilometers from the origin where they refract back to the solar surface over the succeeding hour. Computational seismic holography applied to these surface signatures shows a relatively compact source distribution (Donea, Braun \& Lindsey 1999; Donea et al. 2006; Moradi et al. 2007; Martínez-Oliveros et al. 2007) closely associated with both hard X-ray and white-light emission (Donea \& Lindsey 2005)

Kosovichev \& Zharkova (1998) suggested that the initial source of flare acoustic transients was impulsive thick-target heating of the chromosphere by high energy particles beams (Zharkova \& Kobylinskii 1993). This suggestion was strongly reinforced by Donea \& Lindsey (2005), who found that the acoustic sources were closely co-spatial with hard X-ray emission observed by RHESSI a result confirmed also by Kosovichev (2006a,b,c) and Donea et al. (2006).

We study the structure of some of the most conspicuous seismic transients produced by solar flares. The flares reveal a complex morphology of sources in the egression power maps and also in X-rays. Where RHESSI data available we have investigated the correlation between the motion of the HXR footpoints observed simultaneously with the seismic signatures.

\section{Motion of the HXR sources in solar flares}

The bulk of hard X-rays (HXRs) is emitted from the footpoints (FPs) of flaring loops by fast electrons precipitating into the chromosphere from an acceleration region in the corona. It is well known that the standard model of a flare (Kopp and Pneuman, 1976) predicts an increasing separation of the FP sources as new field lines reconnect at higher and higher altitudes. 
Somov et al. (2005) characterized the motion of the hard X-ray sources by their change of position relative to the simplified magnetic neutral line (SNL). They found that the sources may move perpendicular or parallel to the SNL, in some cases they noted a movement that is a combination of both.

Perpendicular motion (Type I):In some cases the HXR sources appear to have a perpendicular motion to the SNL, showing a separation of the footpoints. Sometimes the motion is not completely perpendicular to the SNL as the direction of motion strongly depends of the magnetic field configuration. The velocity of separation of the footpoints is also proportional to the rate of magnetic reconnection.

Parallel motion to SNL(Type II and III):Somov et al. (2005) defined the Type II as being the anti-parallel motion of the XHR sources, with the FP separating from each other. This motion is attributed to relaxation effects of the non-potential magnetic field. Type III is also parallel, but in this case the footpoints move in the same direction. This possibly indicates that the particle acceleration region in the corona is also moving parallel to the SNL.

\section{Observations and Results}

In order to detect seismic events from flares we have used computational seismic holography (Lindsey \& Braun 2000) to produce maps of the seismic sources at $6 \mathrm{mHz}$. This technique is a phase-coherent reconstruction of acoustic waves observed at the solar surface into the solar interior to render stigmatic images of subsurface sources that have given rise to the surface disturbances. The morphology at the photospheric level of the seismic source detected so far is complex.
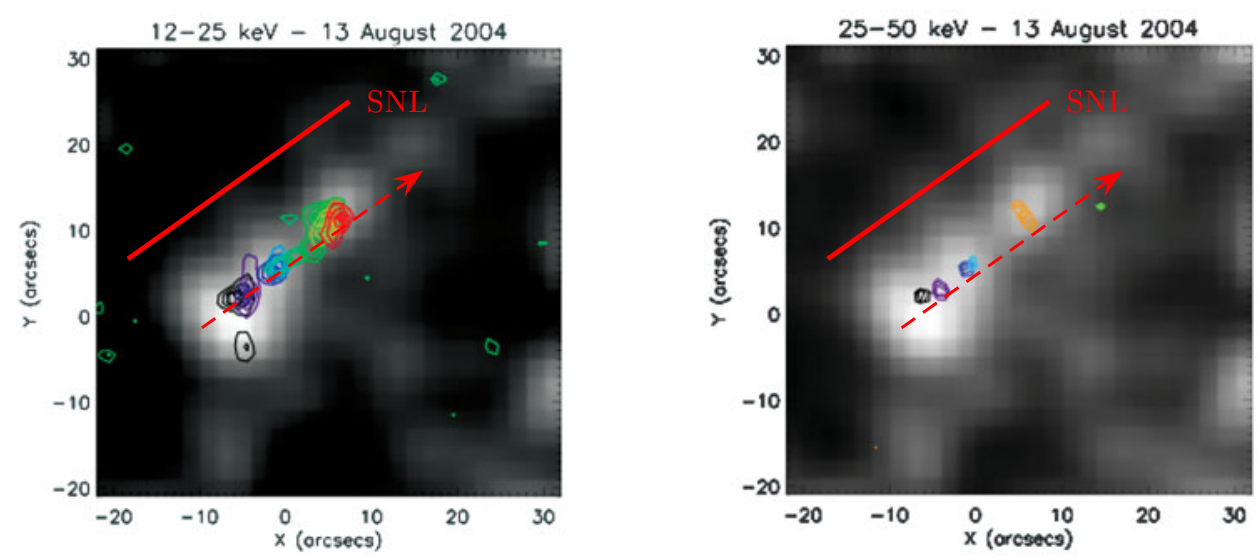

Figure 1. Temporal evolution of the HXR footpoint at $12-25 \mathrm{keV}$ (left frame) and 25-50 keV (right frame). The background image represents the $6 \mathrm{mHz}$ egression power map of the seismic emission produced by the X1.0 solar flare on 13 August 2004. The level countours represent $50 \%, 70 \%$ and $90 \%$ of the maximum of the $15-25 \mathrm{keV}$ flux; $60 \%, 75 \%$ and $90 \%$ of the maximum at $25-50 \mathrm{keV}$ and $70 \%, 80 \%$ and $90 \%$ of the maximum at $25-200 \mathrm{keV}$. The observed times are: 18:11:00 (black), 18:11:30(dark blue), 18:13:00 (blue), 18:13:30 (light blue), 18:14:30 (green), 18:15:30 (green), 18:16:30 (yellow), 18:17:30 (red) for each frame

In many instances the egression-power signature of flare is dominated by a single relatively-compact source. However, some acoustic sources, in microscopic detail, frequently show 2-3 narrow kernels stacked together, separated by intervening nodes. Donea, Braun \& Lindsey (1999) suggested that this was the result of interference caused by rapid 
motion of the source in the direction along which the kernels were stacked, i.e. perpendicular to the nodes that separated the kernels. This proposition was strongly supported by Donea \& Lindsey (2005) who found a similar stacked-kernel structure in the acoustic sources of the flares of 2003 October 28 and 29, for which the motion of the HXR sources were indeed aligned accordingly with the stacks.

The connection between the kernel structure of the seismic sources and the motion of the HXR emission of the Halloween solar flares (Donea \& Lindsey 2005) has inpired us to study other acoustically active flares, with the aim of understanding the dynamics of the seismic transients at the photospheric level. Here we present two kernelled seismic sources from the 13 August 2004 and 15 January 2005 solar flares.

\subsection{August 2004}

On 13 August 2004 at 18:10 UT GOES reported a X1.0 solar flare produced in AR10656. The flare started at 18:07 UT, reached maximum at 18:12 UT and ended at 18:15 UT. We synthetized the RHESSI raw data using the MEM-SATO algorithm (Sato et al. 1999) to obtain images of the $12-25,25-50$ and $50-200 \mathrm{keV}$ X-ray footpoints with a cadence of 10 seconds (Figure 1). The data shows X-ray footpoint movement parallel to the SNL.

The RHESSI HXR observations show footpoint emission above $12 \mathrm{keV}$ originating from the two ribbons of a magnetic arcade (Krucker et al. 2003 reported a similar behaviour for the 23 July 2002 solar flare; we also have reported the detection of a seismic event from this flare). Contrary to the simple reconnection models the direction of motion is roughly along the magnetic neutral line and not perpendicular to it. This indicates that the reconnected magnetic field lines are highly sheared, a condition which seems to exist in acoustically active solar flares. The continuous motion along a straight line suggests that footpoints of newly reconnected loops are close to each other. We find an excellent correlation between the direction of motion of the HXR sources and the main axis of the kernelled structure of the seismic source.

\subsection{January 2005}

The seismically active flare of 15 January 2005 represents a very interesting seismic case, starting at 00:22 UT, reaching a maximum at 00:43 UT and ending at 01:02 UT. This flare produced the most conspicuous and powerful sunquake detected so far (Moradi et al. 2007). Using the same synthesis process, described before, we found that the RHESSI $12-25 \mathrm{keV}$ and 25-50 keV contour plots (Figure 2) do not present a dominant direction
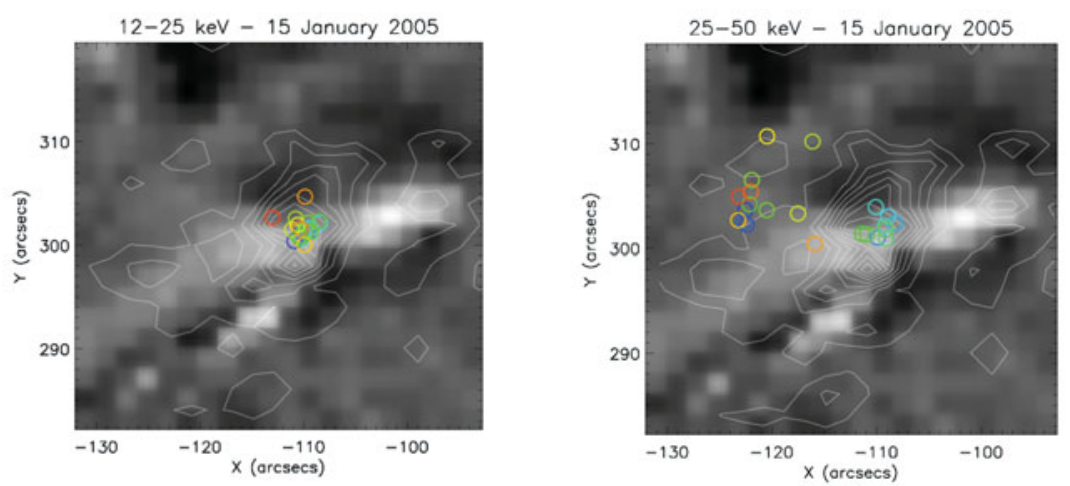

Figure 2. Temporal evolution of the HXR footpoint at $12-25 \mathrm{keV}$ (left frame) and 25-50 keV (right frame). Background image represents the flare signature seen in the Doppler difference frames at the maximum of the flare (00:44 UT) and the white contours shows the $6 \mathrm{mHz}$ egression power map of the seismic emission. Circles represent the centroids of the RHESSI HXR sources. 
of motion, but always are localized on a small region were also detected the main $6 \mathrm{mHz}$ egression power kernel.

\section{Conclusions}

We have studied the spatial properties of the hard X-ray emission of seismically active flares. The majority of the studied egression power signatures present some kind of structure, that can be a collection of kernels or a single kernel accompanied with clear acoustic wings or halos. We found an empirical relation between the direction of motion of the HXR footpoints and the spatial alignment of the egression power kernels, being the parallel motion of type III the most common type of motion. This observations are followed by simulations of the events in a future paper by Donea, Martinez-Oliveros and Lindsey (in preparation).

\section{Acknowledgements}

Juan Carlos Martínez-Oliveros thanks the Astronomical Society of Australia (ASA) and International Astronomical Union Symposium 247 (IAUS247) for travel support.

\section{References}

Donea, A.-C., Beşliu-Ionescu, D., Lindsey, C. \& Zharkova, V. V. 2006, Solar Phys. 239, 113

Donea, A.-C., Braun, D. C \& Lindsey, C., 1999, Astrophys. J. 513, L143

Donea, A.-C., \& Lindsey, C., 2005 Astrophys. J. 630, 1168

Kopp, R. A., Pneuman, G. W., 1976, Solar Phys. 50, 85.

Kosovichev, A. G. \& Zharkova, V. V. 1995, Proc. $4^{\text {th }}$ SOHO Workshop (ESA SP-376) p. 341.

Kosovichev, A.G., Zharkova, V.V., 1998, Nature 393, 317

Kosovichev, A.G., 2006, Proc. SOHO 18/GONG 2006/HELAS Conf. (ESA SP-624) p. 134.

Kosovichev, A.G., 2006, Solar Phys. 238, 1

Kosovichev, A.G., 2006, ASP Conf. Ser. V. 354, p. 134.

Krucker, S., Hurford, G. J. and Lin R. P., 2003, Astrophys. J. 595, L103

Lindsey, C., \& Braun, D. C., 2000, Solar Phys. 192, 261.

Martinez-Oliveros, J.C., Moradi, H., Besliu-Ionescu, D.,Donea A.-C, Cally, P., 2007, Solar Phys. 245, 121

Moradi, H.; Donea, A.-C.; Lindsey, C.; Besliu-Ionescu, D.; Cally, P. S.,2007, Mon. Not. Roy. Astron. Soc. 374, 3, 1155

Sato, Kosugi, Makishima, 1999, Pub. Astron. Soc. Japan 51, 127

Somov, B.V., Kosugi, T., Bogachev, S.A., Sakao,T. and Masuda, S., 2005, Adv. Spa. Res. 35, 10,1700

Zharkova, V.V., \& Kobylinskii, V.A., 1993, Solar Phys. 143, 249. 